العدد السادس عشر

مجلة العمارة والقنون

دراسة الأساليب الفنية الحديثة لامج الرسوم المتحركة ثنائية الأبعاد وثلاثية الأبعاد وفقاً لمنطلبات أفلام الرسوم المتحركة

Studying new techniques of composing 2D animation with 3D animation required for Animation Film production

\author{
م.د/ أيمن رأفت إسماعيل الجندي \\ مدرس دكتور بقسم الميديا والتحريك بكلية الفنون التطبيقية ــ الجامعة الألمانية بالقاهرة
}

Dr. Ayman Raafat Ismail Elgndy

Lecturer at Media Department - Faculty of Science and Arts - German University in Cairo

ayman.elgndy@guc.edu.eg

المقدمة

الرسم المتحرك هو تسلسل من الصور، أو الإطارات، التي تُعرض عبر الوقت. ويختلف كل إطار بعض الثيء عن الإطار الذي يسبقه، مما يُنشئ خداع الحركة أو أبي تغييرات أخرى عند عرض الإطارات بتسلسل سريع ، وإن الحركة المقصودة هي معني واسع وعام يمتد ليشمل أي تغيير يطر أ علي الصورة, وقد بدأ ظهور ذلك المصطلح بعد ظهور أفلام الكارتون التي أنتجتها شركة والت ديزني , مثل سلسلة الأفلام الثهيرة توم أند جيري , و غيرها فهي قائمة أساسا علي الرسوم المتحركة ونظرية الحركة وتنوعت أثنكال الحركة منها التحريك ثنائي الأبعاد و التحريك ثلاثي الأبعاد ومن أكثر الأثياء التي تسبب مشاكل في التحريك هي الدمج بينهم و صناعة شكل الحركة لتكون أقرب للواقع بناء علي منطلبات أفلام الرسوم المتحركة الني كانت صعب تنفيذها بالماضي مما جعلها موضع نطوير و إضافة في برامج التصديم و التحريك و المعالجة للصورة الثابتة و المتحركة . . .

فان عملية دمج وربط كل من تحريك الرسومات ثنائية الأبعاد والمجسمات ثلاثية الأبعاد وتحريكها داخل فراغ ثلاثي الأبعاد مع إضافة كاميرا متحركة تربط المشهد وتتعامل مع كافة الرسوم والمجسمات المتحركة داخل المشهد في نفس الفر اغ مع إمكانية محاكاة خصائص كامير ا بر امج التحريك الرقمي للكامير ا الحقيقية سواء كانت تغيير أنواع العدسات او فتحة وسرعة الكاميرا مع إضافة تأثثرات عمق الميدان ومتغيرات السرعة و أيضا إمكانية التحكم في حركات الكاميرا داخل المشهد طبقا للسيناريو والرؤية الإخراجية وتتنافس الإصدارات الحديثة من برامج التحريك و التركيب و الدمج

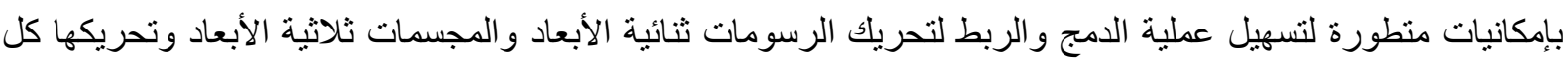
داخل الفراغ ثلاثي الأبعاد بشكل أفضل ومن أشهر تلك البرامج ( أدوبي أفتر أفكتس ) التابع لشركة أدوبي الأمريكية مستخدما أدوات مساعدة تتيح له تلك الإمكانية ، وبرنامج تون بوم هارموني الإصدار برميم و الذي يتميز بقدرة فائقة علي تنفيذ تللك العملية وبجودة عالية وفيما يلي سنتعرف علي مر احل الانتاج للفيلم المتحرك مع كيفية دمج وربط كل من الرسومات ثنائية الأبعاد والمجسمات

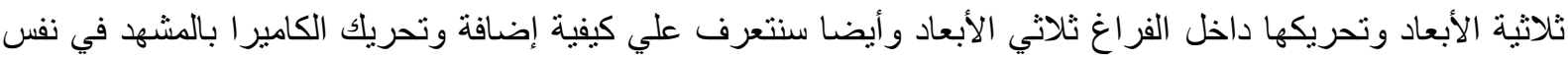
فر اغ مع إمكانية محاكاة خصائص الكامير اللكامير ا الحقيقية. كلمات مفتاحية: الرسوم المتحركة ؛ ثنائية الأبعاد ؛ ثلاثية الأبعاد ؛ دمج. 
Abstract:

Animation is a sequence of frames played per seconds and started to be a successful production when Walt Disney started animating his famous series like Tom and Gerry, But the problems started when the animated films started using digital animation and advanced techniques and the 3D animation using 3D objects and Camera movements have got a big market of animation production and then animated film starts asking about composing 2D animation with 3D animation, all these needs forced animation software to start to develop its options so that it can serve animation films and animators requirements starting by Adobe After effects which offers great results in motion graphics, animation and composting, but also Toon boom have released harmony premium The most powerful software ever created for animation production so that we can Access to a complete toolset for traditional frame-byframe animation. Or Build simple to advanced cut out rigs with specialized tools. Master controllers save time when animating sophisticated rigs.

Also we can Import 3D Objects as Once we have exported a model using a 3D authoring software, we can import it into a 2.5 Scene and integrate it to set up your $2 \mathrm{D}$ animation scene. we have the possibility to convert our $3 \mathrm{D}$ models to the *.fbx format compatible to be imported to Harmony. This will allow us to render 3D files with the associated textures without the need to copy over the textures manually .

Using multiplane space is a 2.5 Animation where we can move layers forward and backward on the Z-axis. Latest Animation softwares like toonboom harmony brings us a true threedimensional space where we can actually rotate your camera and layers on all axes. This lets us perform a 360 degree rotation around elements, create a floor and even build sets!

Once we have a 3D set, the exciting part is to do a camera move in it. Once we know how to do this, we can enjoy the delights of working in 3D space and traveling inside with $2 \mathrm{D}$ or $3 \mathrm{D}$ animations together.

We can change the near and far clipping planes of the camera. The near plane is the point on the camera cone where the camera is located. The far clipping plane is the far end of the camera cone. Nothing outside that range is visible. This is useful when dealing with $3 \mathrm{D}$ elements and 3D sets.

And finally these latest solutions help us to animate 3D models and their individual parts directly while integrating $3 \mathrm{D}$ elements with $2 \mathrm{D}$ characters.

Keywords: animation; 2d; 3d; compositing.

$$
\text { من منكلة البحث: }
$$


دراسة الأساليب الفنبة الحديثة لامج الرسوم المتحركة ثنائية الأبعاد وثلاثية الأبعاد مع إضافة وتو افق حركات الكاميرا بين العناصر ودون أي فقد في الجودة . تساؤلات البحث: • ما هي البرامج الحديثة المستخدمة في لدمج الرسوم المتحركة ثنائية الأبعاد وثلاثنية الأبعاد؟

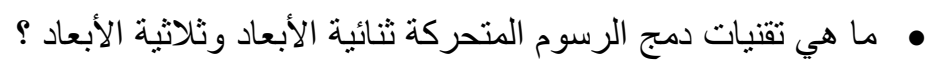

يسلك الباحث المنهج الوصفي التحليلي للأساليب الفنية الحديثة لدمج الرسوم المتحركة ثنائية الأبعاد وثلاثية الأبعاد مع إضافة وتو افق حركات الكامير ا. فروض البحث: إذا تم اتباع الأساليب الفنية الحديثة لدمج الرسوم المتحركة ثنائية الأبعاد وثلاثية الأبعاد سوف يؤدي ذلك الي فيلم رسوم

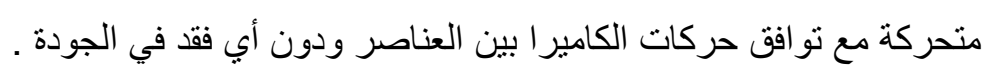

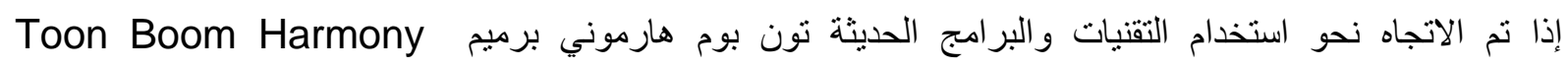
الإصدار الأخير فسوف يساهم ذللك في سرعة الإنتاج داخل برنامج واحد دون استخدام برامج للامج . ا ـ ا الانتاج الرقمي للاسوم المتحركة : تطورت عملية انتاج الرسوم المتحركة مع تطور التكنولوجيا الرقمية وظهور برامج وأدوات حديثة ومتطورة في عمليات

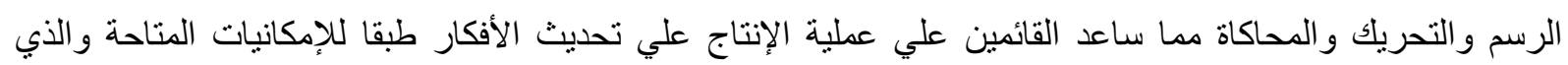
تطلب إعادة هيكلة المنظومة الإنتاجية كما هو مبين بالمخطط التالي: 


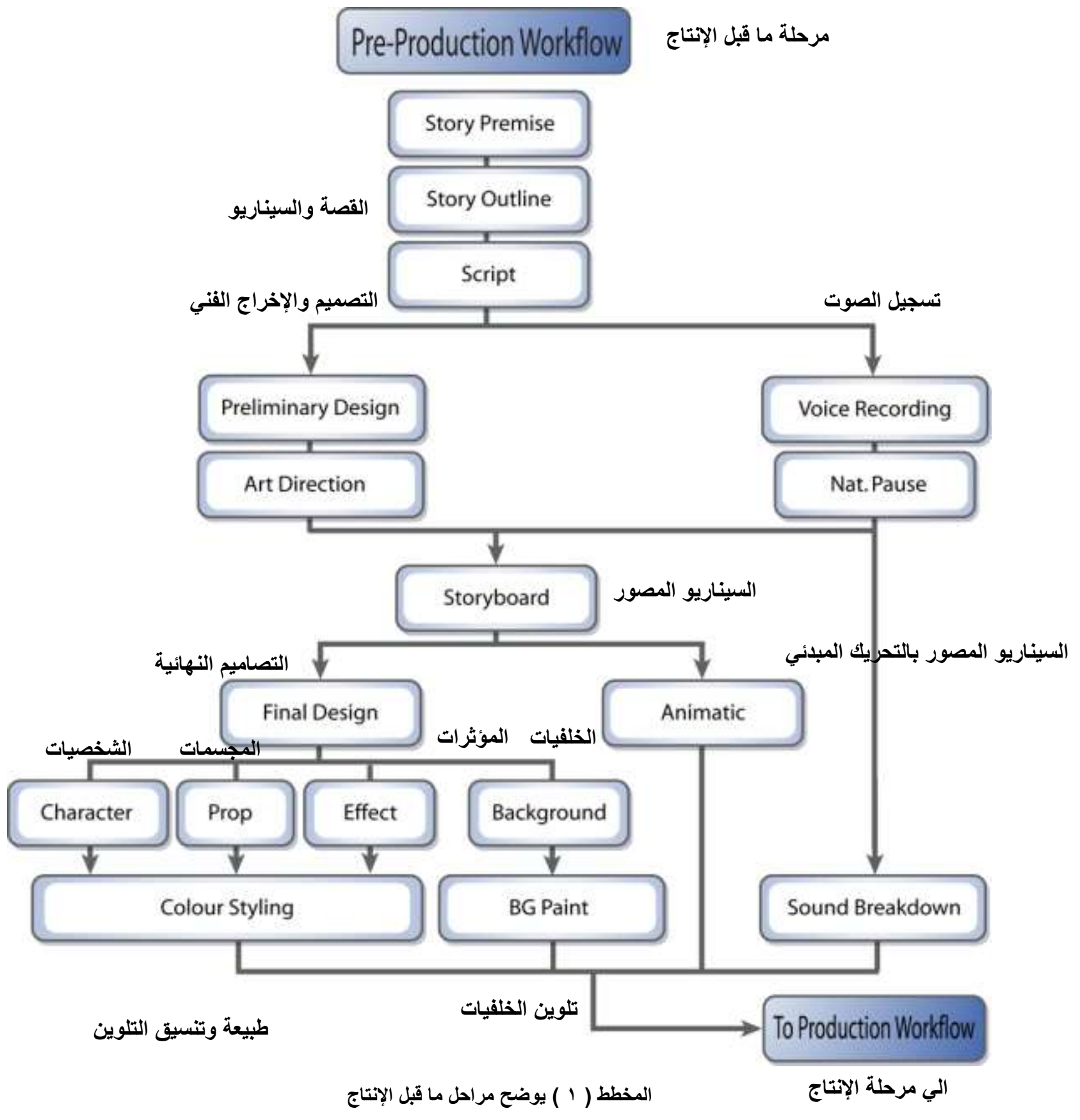


مرحلة الإتتاج

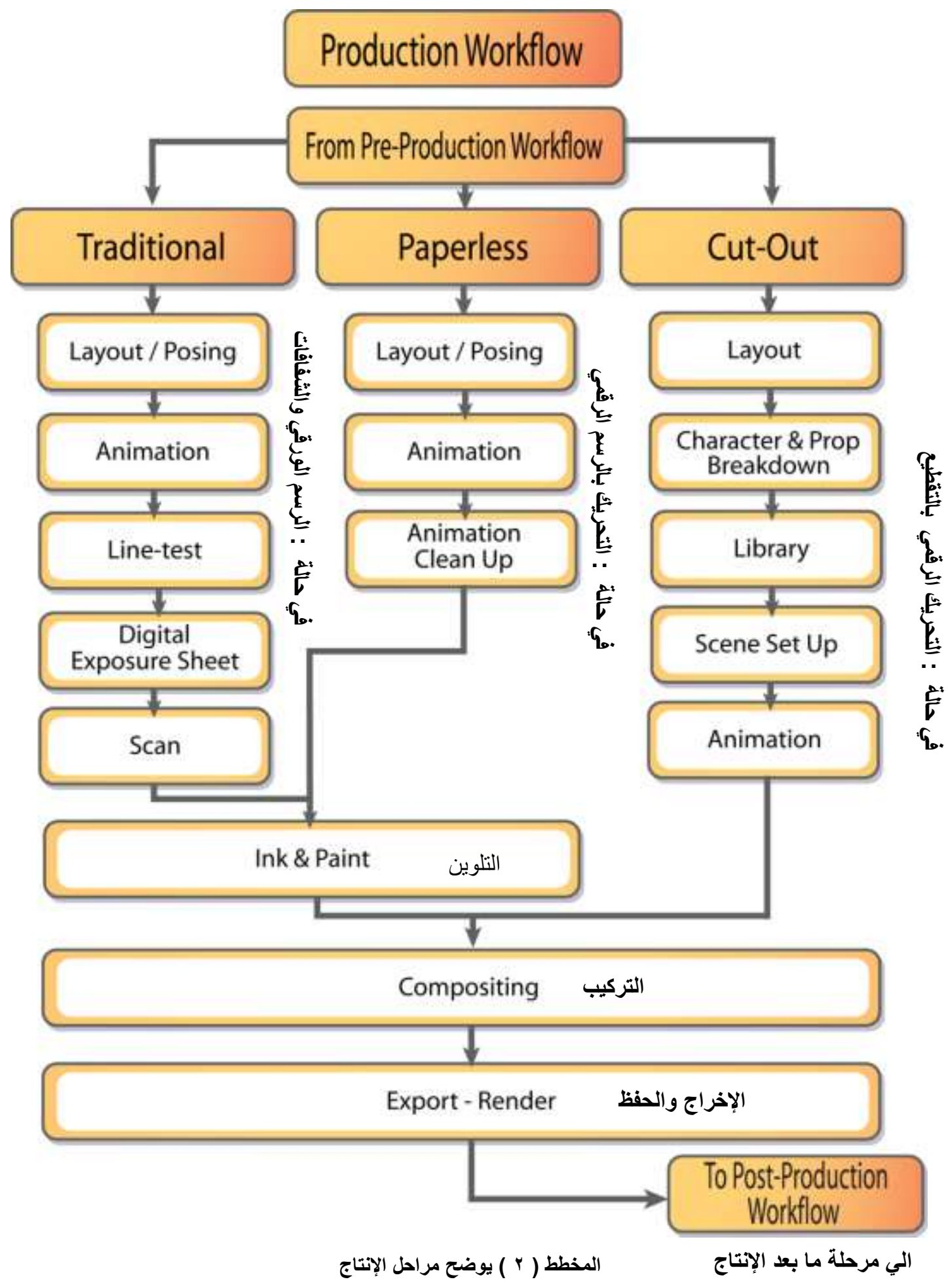




\section{Post-Production Workflow}

\section{From Production Workflow}

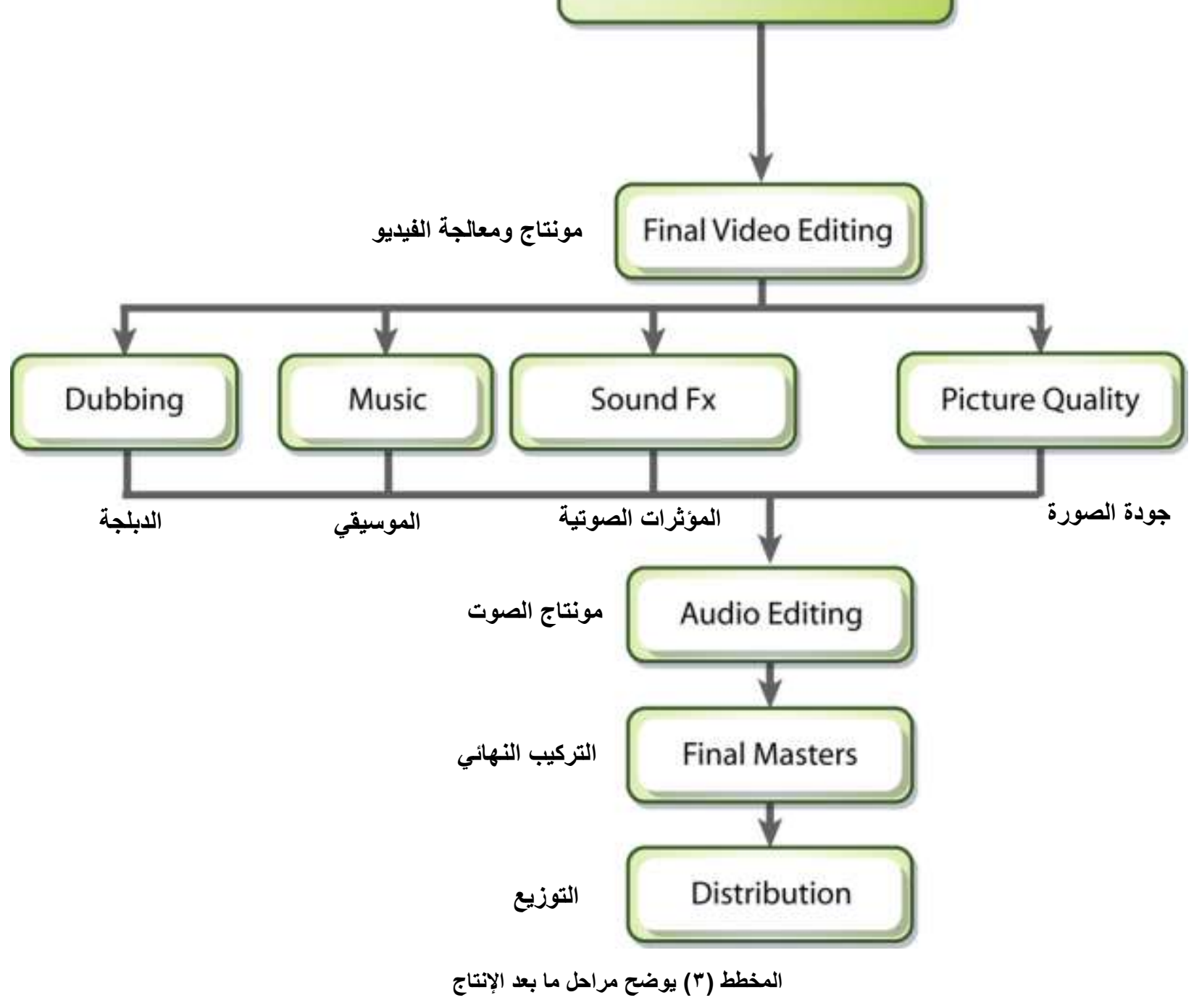

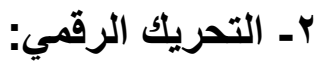

تطورت عملية انتاج الرسوم المتحركة مع تطور التكنولوجيا الرقمية وظهور برامج وأدوات حديثة ومتطورة في عمليات الرسم و التحريك و المحاكاة مما ساعد القائمين علي عملية الإنتاج علي تحديث الأفكار. r ـ ا التحريك الرقمي ثنائي الأبعاد : و هي عملية التحريك معتمدا علي الرسم وتتابع الحركة بالرسومات الابعاد : لمتتالية في المدة المحددة لتنفيذها وتعتمد تلك الطريقة علي مهارة الرسم والتوقيت للحركة وتستغرق وقت ومجهود كبير لإنهاء الرسم ونقله علي الكمبيوتر وإعادة تجميع

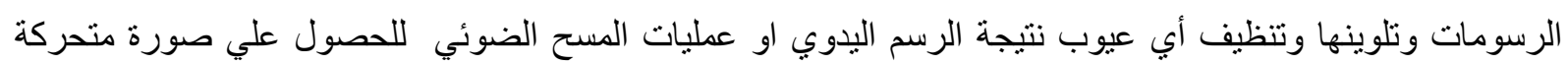
جيدة كما هو موضح بالثكل ( ) 


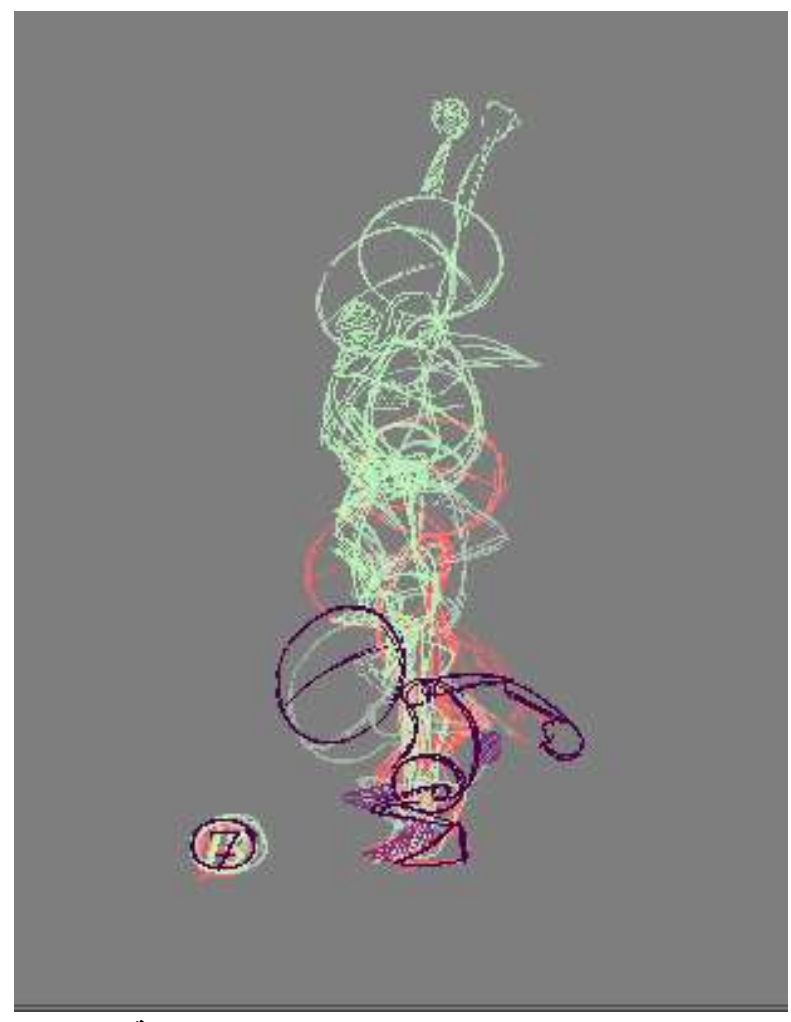

شكل ( ) يوضح تتابع الرسومات لتكوين الحركة

r ـ ا ـ ا التحريك ثنائي الأبعاد بالرسومات الرقمية : و هي عملية الرسم و التحريك داخل برنامج بالكمبيوتر معتمدا أيضا علي الرسم وتتابع الحركة بالرسومات المتتالية لكن

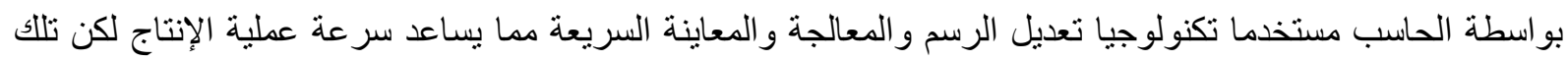
الطريقة ماز الت تعتمد علي مهارة الرسم وتخيل الحركة والتوقيت الجيد للحركة وتستغرق وقت اقل ونتائج أدق بكثر من

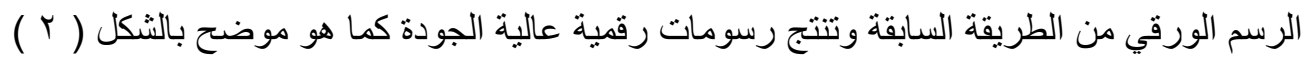

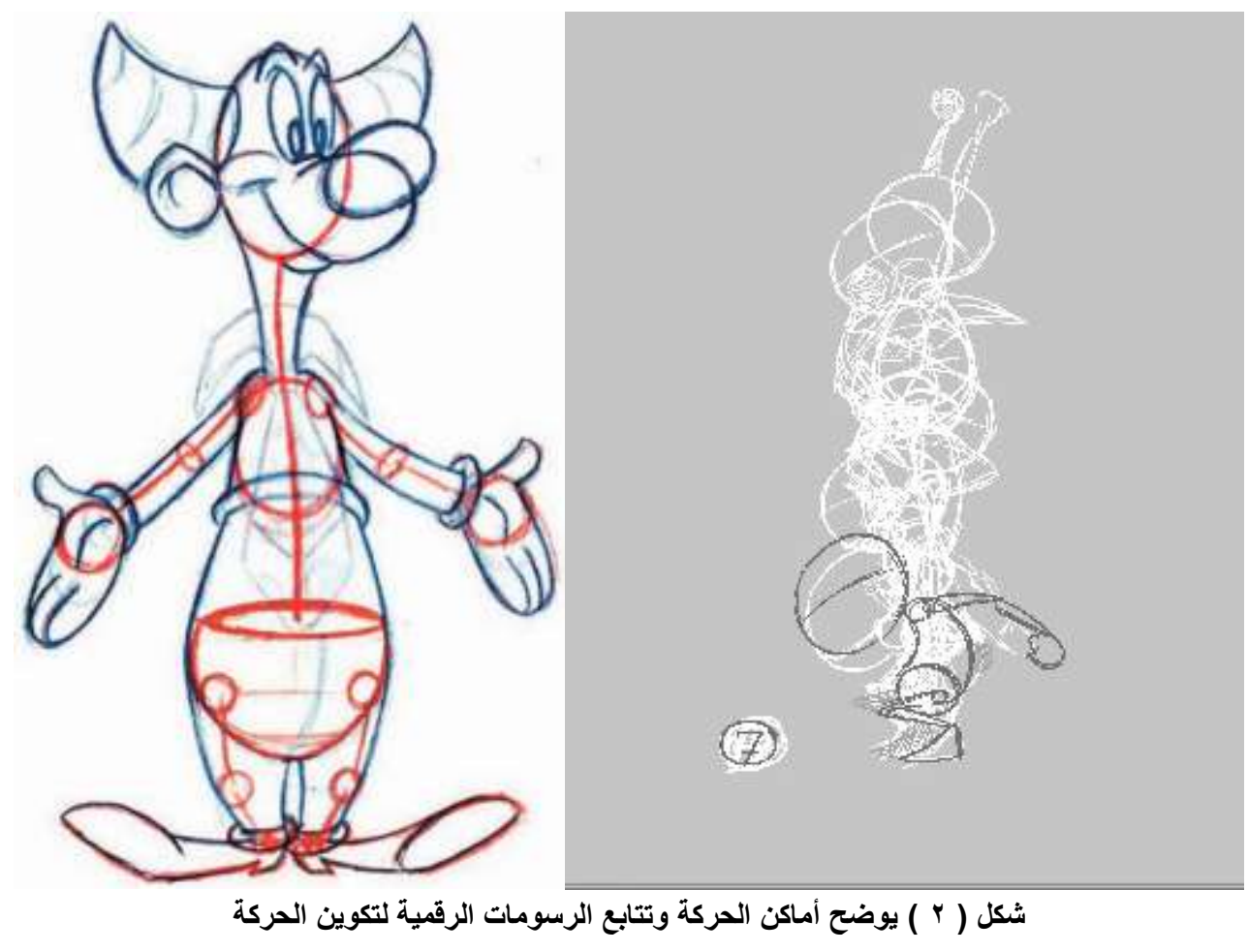




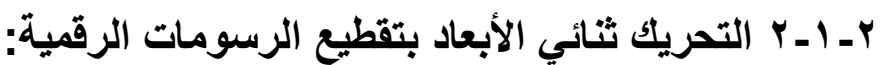

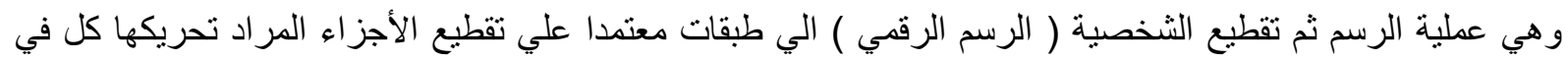

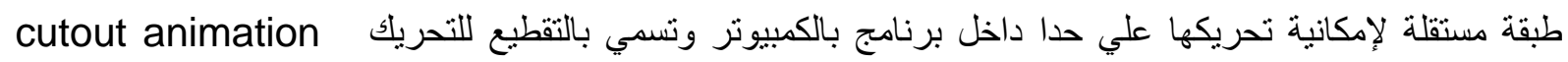

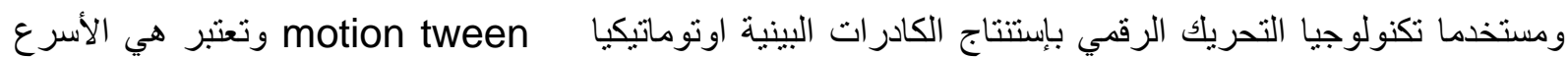
علي الإطلاق في عملية إنتاج الرسوم المتحركة وتتطلب نللك الطريقة تخيل الحركة و التوقيت الجيد لها وتستغرق وقت اقل

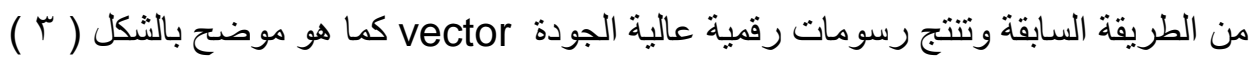
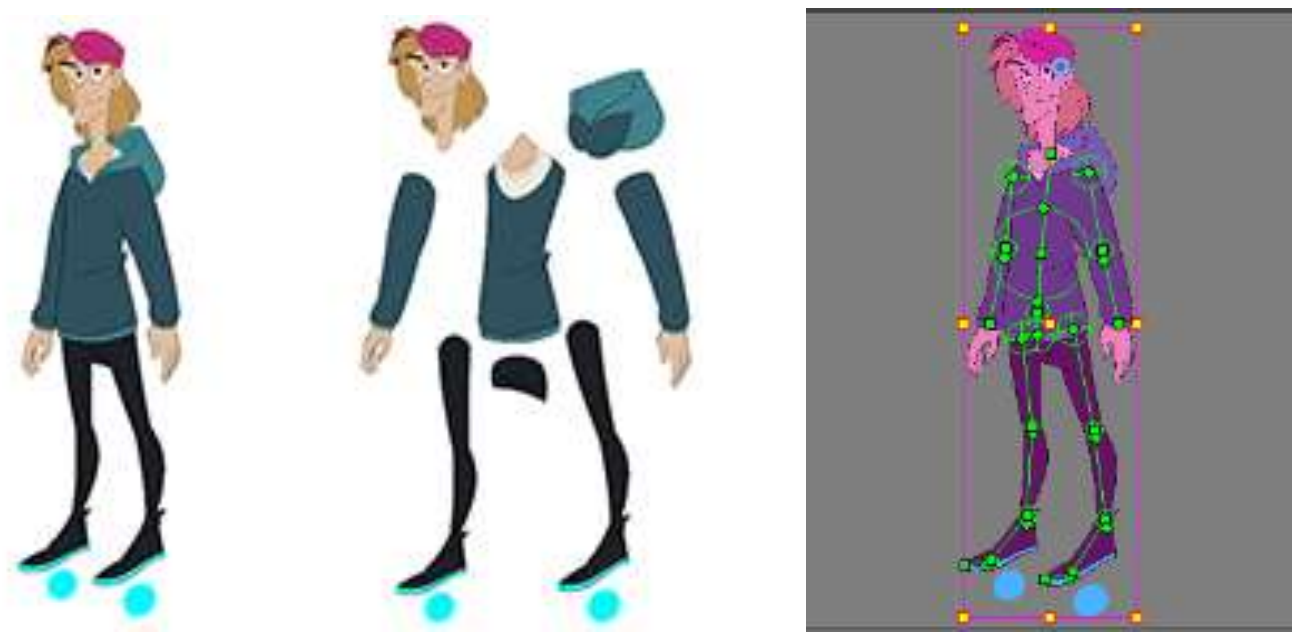

شكل ( r ) يوضح نظام التقطيع للرسم للتحريك

وبإستخدام خاصية العظام bones في تحريك طبقات المفصلات بالثخصيات نحصل علي نتائج جيدة خاصة لتفهم نظام العظام bones بخو اص الحركات الكاينيماتكس العكسية بجسم الانسان inverse kinematics و التي تعطي حركة الاستجابة الاوتوماتيكية لأجزاء الجسم مع إمكانية التحكم بدرجات ومدي الإستجابة لكل عظمة داخل جسم الثخصية و

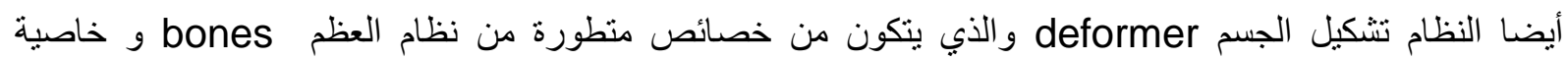
المنحنيات curves و التي تعتبر هي الأحدث في تحريك وتتككيل الأجسام دون الحاجة الي تقطيع الجسم ككل، بل أن الأجز اء المرتبطة يمكن رسم العظم عليها لتتعامل معها كأنها مقطعة مما يوفر وقت ومجهود تقطيع الطبقات كما هو مبين

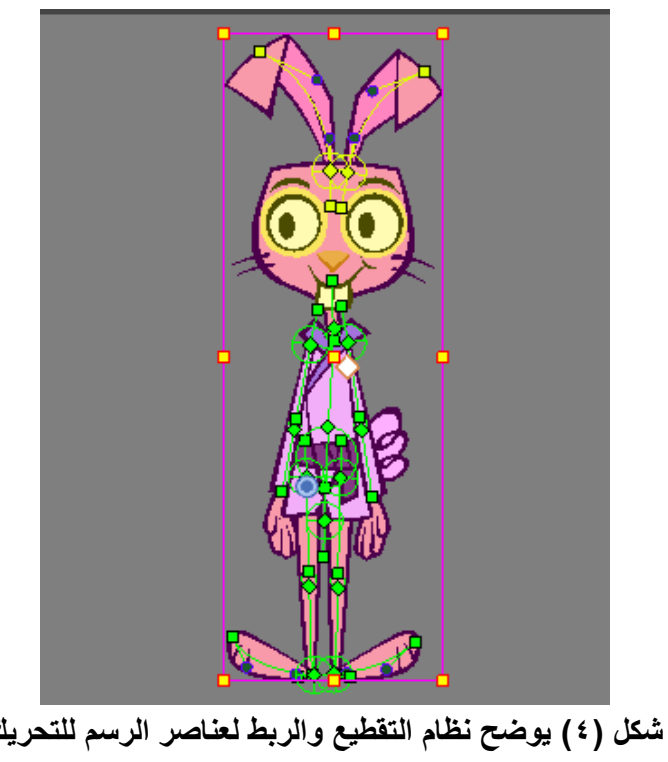


وتعتبر عملية ربط أجز اء طبقات الجسم طبقا لتأثير كل طبقة في الأعلى parent علي الطبقات اسفلها child باستخدام

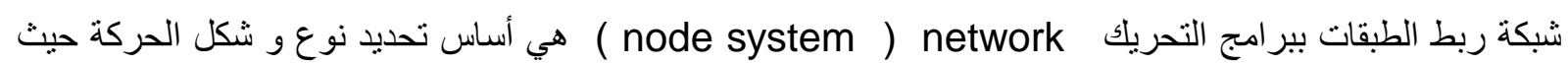

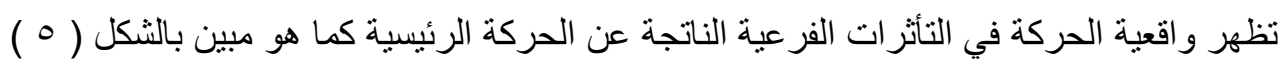

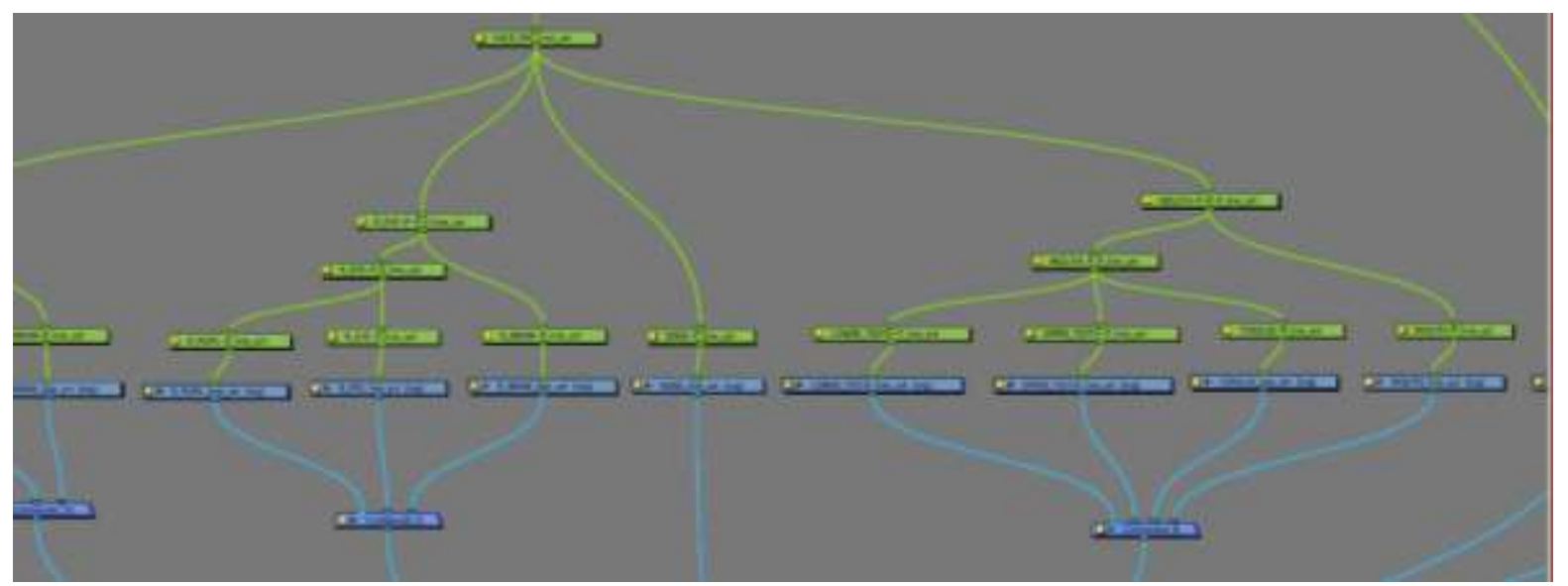

شكل ( • ) يوضح نظام شبكة ربط طبقات عناصر الرسم للتحريك

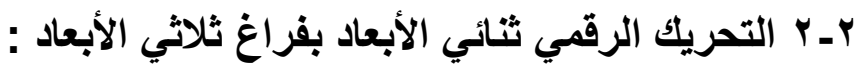

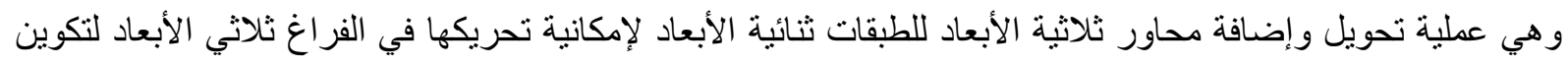
مشهر يحتوي علي عمق و ابعاد ثلاثية الأبعاد لكن بإستخدام رسومات و طبقات ثنائية الأبعاد يمكن من خلالها استغلال

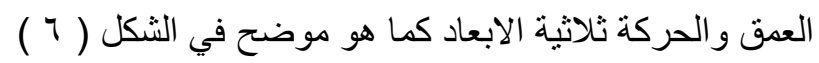

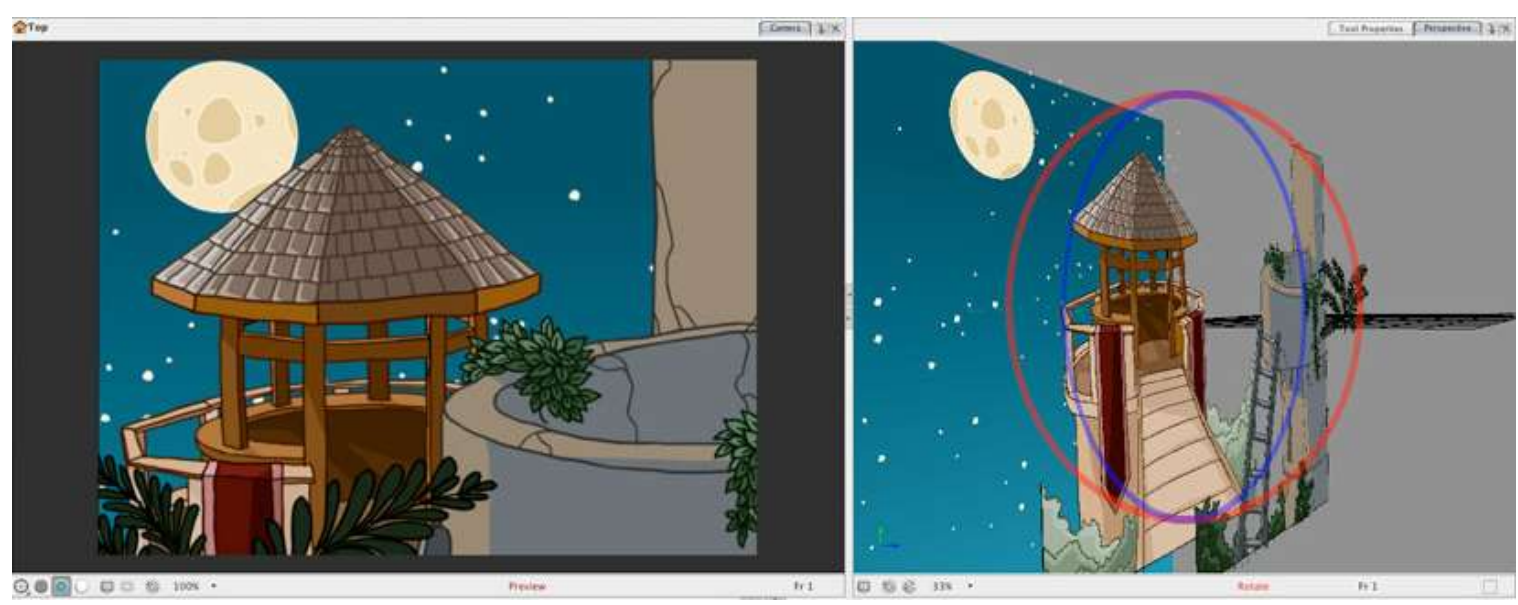

شكل ( " ) يوضح الطبقات ثنائية الأبعاد داخل فراغ ثلاثي الأبعاد

وتعتبر خاصية تحريك الطبقات و الرسوم ثنائية الأبعاد بمحاور ثناثية الأبعاد X Y Z داخل الفراغ هي من النوع 2.5 D و التي يمكن من خلالها تصميم وتوزيع اضاءة علي الطبقات والحصول علي تأثير الظلال مما يساعد علي تجسيم

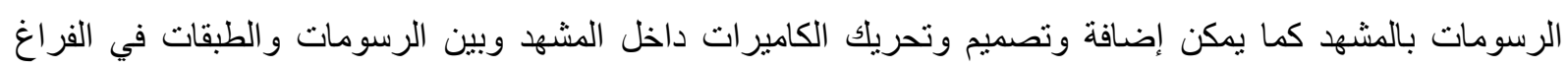

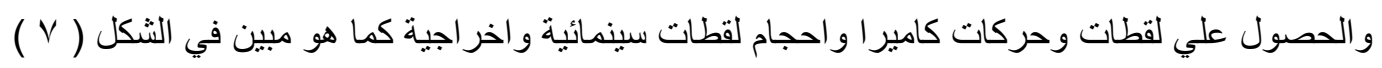




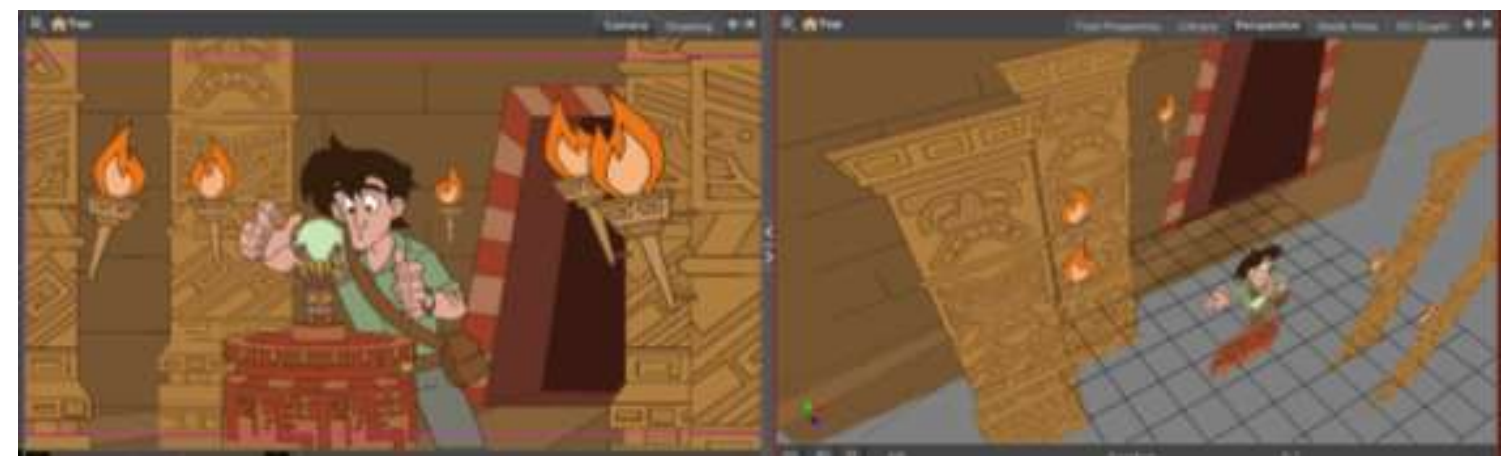

شكل ( V ) يوضح الطبقات ثنائية الأبعاد داخل فراغ ثثلاثي الأبعاد مع حركة الكاميرا

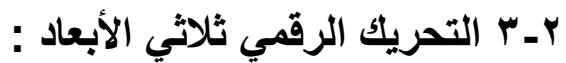

وتتميز الإصدارات الحديثة من برامج التحريك ثنائية الأبعاد مثل أدوبي أفتر أفكتس و تون بوم هارموني برميم بإمكانية

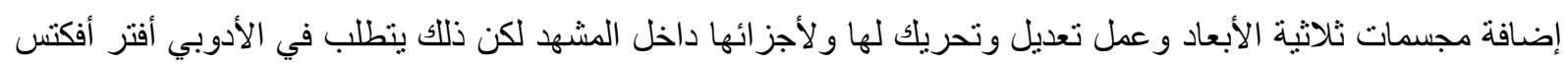

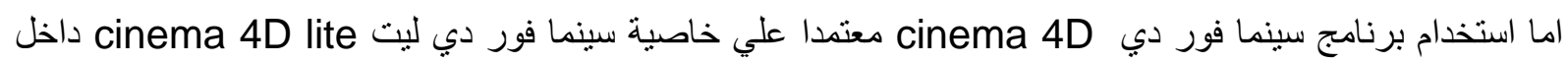
مؤثرات الأفتر افكتس أو إضافة برامج مساعدة للبرنامج مثل سين وير من ماكسون cineware أو بإستخدام إضافة إلمنت Element 3D داخل برنامج الأفنر أفكتس . وعن توم هارموني Toon Boom Harmony فالإصدار بريميم هو المدعم الوحيد لتلك الخاصية و الذي يتميز بنفس بـإن الإمكانيات و يدعم ادر اج الملفات والمجسمات ثناثية الأبعاد داخل المشهد بسهولة مع إمكانية تحريكها ولكي ينم إخراج

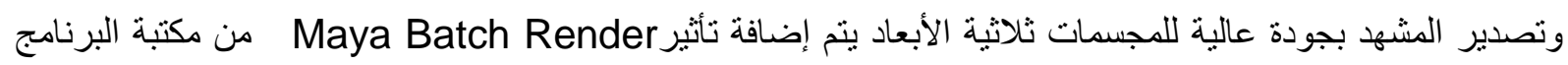

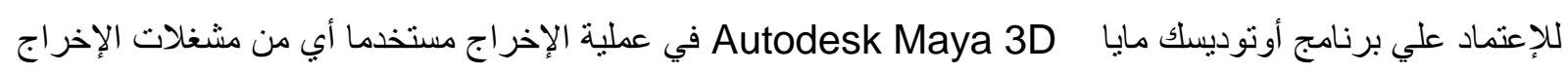
الثهيرة مثل Render Man و Maya software و أو Rental ray وأخبرا Arnold الأحدث لدي أوتوديسك في إخر اج المشهد بجودة عالية كما هو مبين بالشكل ( 1 )

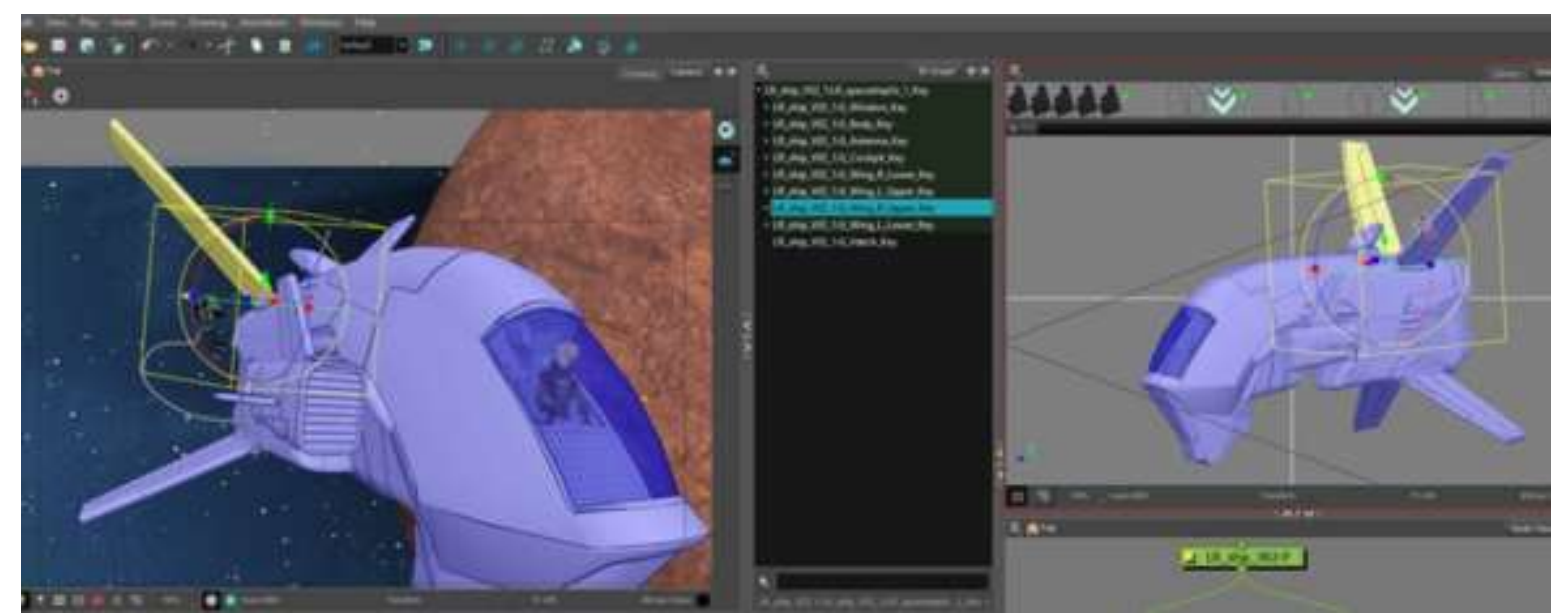

شكل ( ^ ) يوضح المجسم ثلاثي الأبعاد داخل فراغ ثُلاثي الأبعاد

بـ دمج الرسوم ثنائية الأبعاد مع المجسمات ثلاثية الأبعاد :

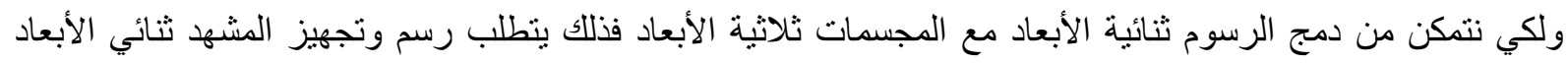
بيرنامج الرسم مثل أدوبي اليستريتور أو تون بوم هارموني مع تحضير و بناء العناصر ثلاثية الأبعاد بيرنامج النمزجة مثل أوتوديسك مايا أو ثري دي ماكس . 
ولكي نتمكن من الدمج فإن برنامج تون بوم هارموني برميوم Toon Boom harmony Premium يعد الأفضل حيث نستطيع رسم وتقطيع و تحريك المجسمات ثنائية الأبعاد ثم تحويها و إضافة محاور ثلاثية الأبعاد لها بفر اغن ثلاثني

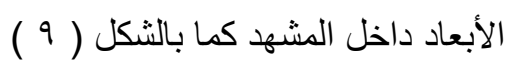

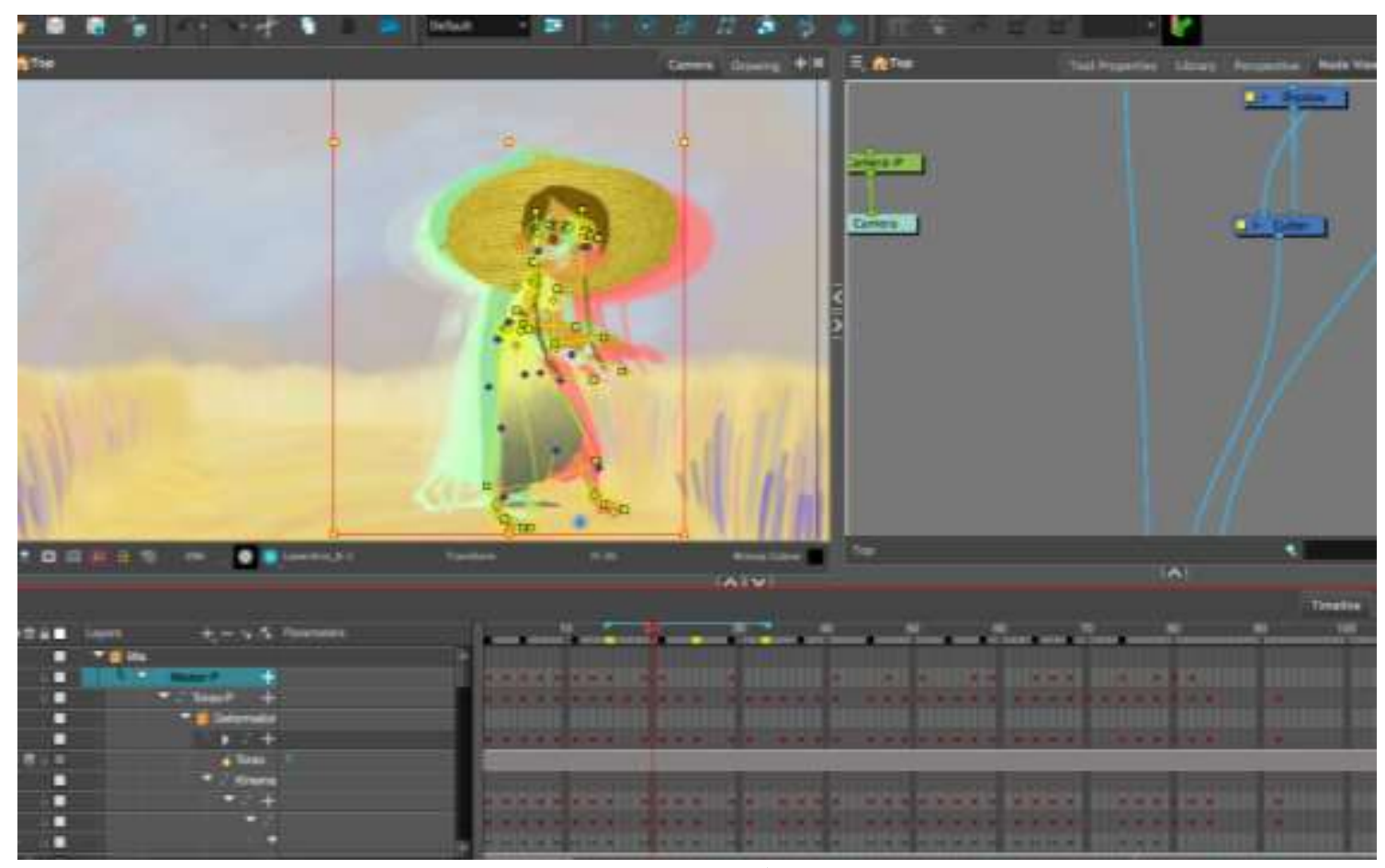

الثكل ( 9 ) من داخل واجهة برنامج تون بوم هارموني الإصدار برميم

ويمكن بعد ذلك ادر اج ودمج المجسمات ثلاثية الأبعاد بصيغة FBX داخل نفس الفراغ بالبرنامج وحينها يكون تم الدمج الرسوم ثلاثية الأبعاد مع المجمات ثلاثية الأبعاد بنفس المشهر و عمل التحريك و التعديل لأي منهم مع إمكانية إضافة

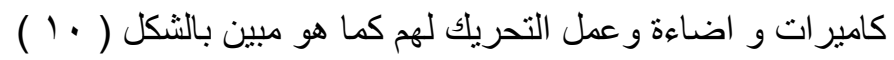

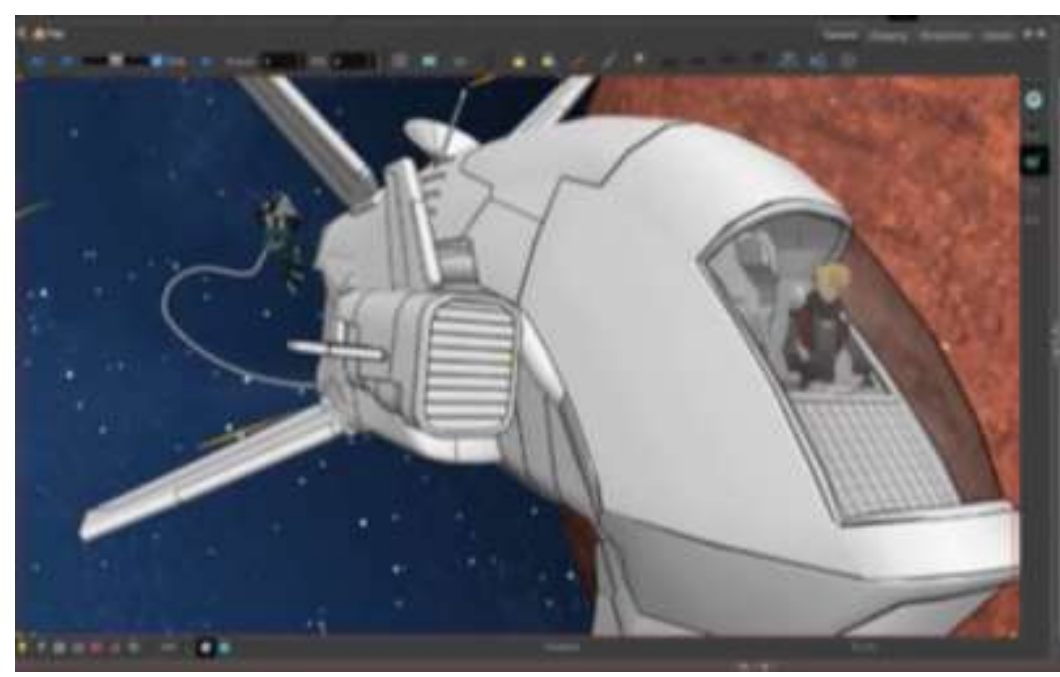

شكل ( · 1 ) يوضح دمج شخصية متحركة ثنائية الأبعاد داخل مجسم ثلاثي الأبعاد 
و هي المرحلة الأخيرة من دمج الرسوم ثنائية الأبعاد مع المجسمات ثلاثية الأبعاد بعد إضافة حركة الكامير ا داخل المشهد حيث يمكننا بإستخدام التأثثر OrthoLock ربط زاوية رؤية وحركة الطبقات ثنائية الأبعاد بإتجاه الكاميرا حيث تتبح زاوية رؤية الطبقات ثنائية الأبعاد حركة الكاميرا داخل الفر اغ ثلاثي الأبعاد كما هو مبين بالثكل ( 11 ( )

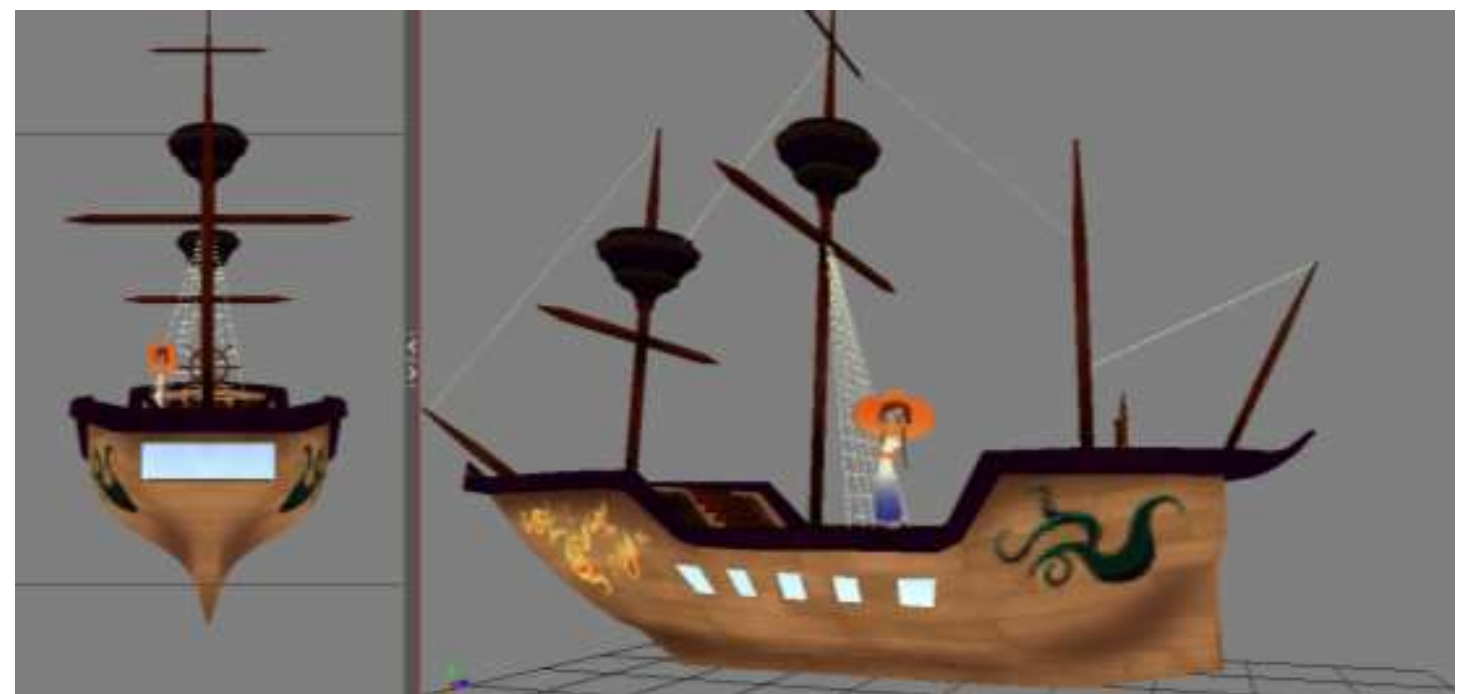

شكل (1 ' ) يوضح دمج شخصية متحركة ثنائية الأبعاد داخل مجسم ثلاثي الأبعاد مع حركة كاميرا

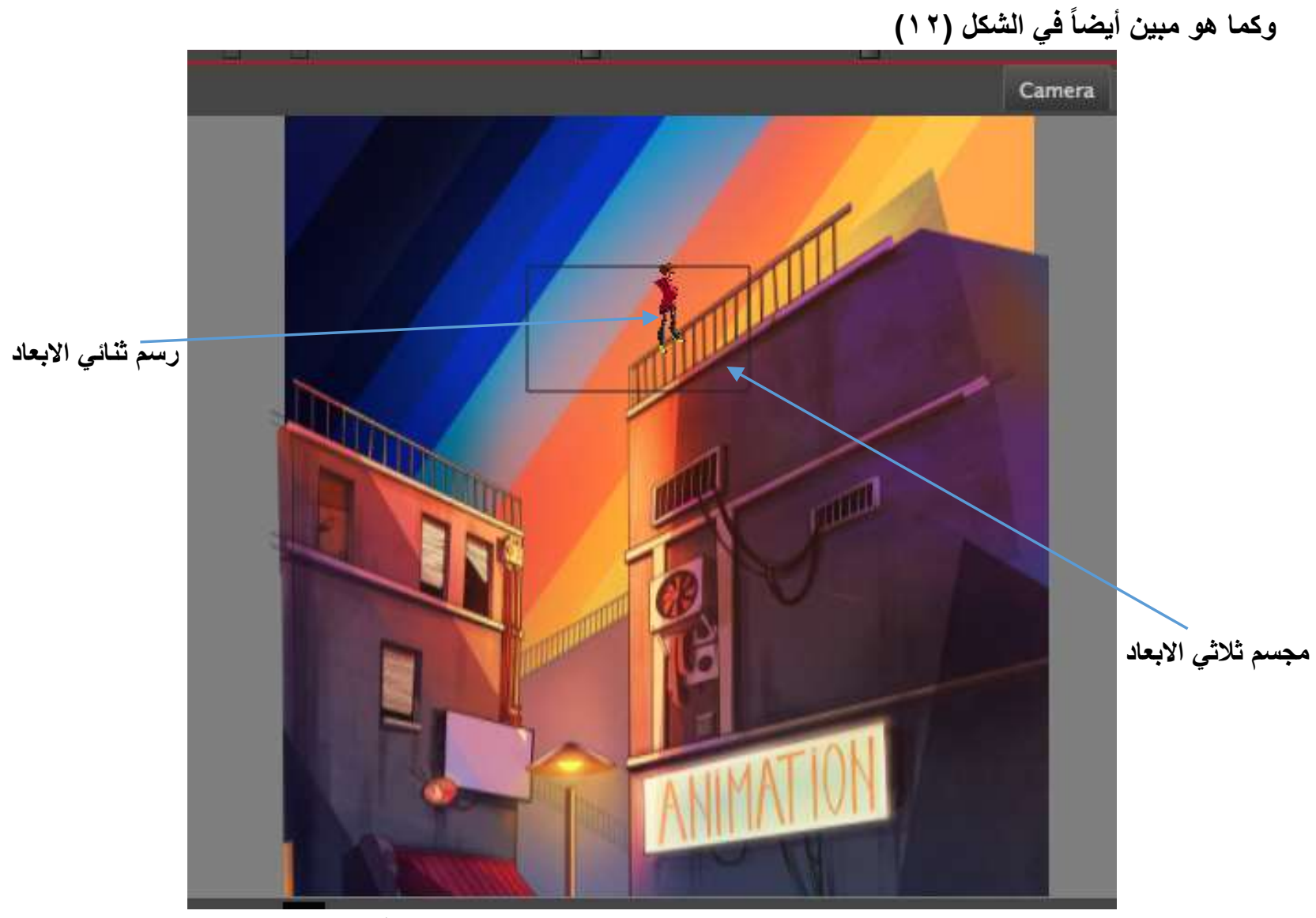

الثكل (Y ) رسم متحرك ثنائي الابعاد مع مجسم متحرك ثلاثي الأبعاد 
1 - التحريك والرسم الرقمي هو الأكثر استخداما في انتاج أفلام الرسوم المتحركة 2 - استخدام كامير ا بر امج التحريك هي الأفضل في جودة الإنتاج من كامير ات بر امج التركيب والدمج 3 - برنامج تون بوم هاموني برمييم هو الأفضل في عمليات تحريك وإخراج مشاهد ثنائية الأبعاد مدمجة بعناصر متحركة ثلاثية الأبعاد 4 - لإنتاج رسوم متحركة ثنائية الأبعاد مدمجة بعناصر منحركة ثلاثية الأبعاد نحتاج البر امج التالية :

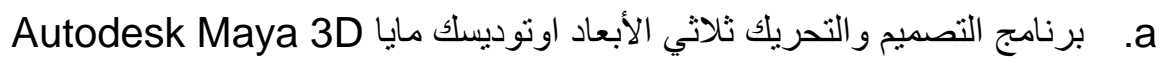

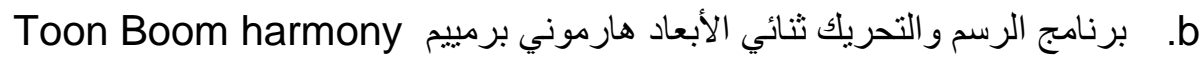
5 - عند استخدام مكتبات او نماذج ثلاثية الأبعاد من بر امج اخري ودمجها بالمشاهد المتحركة ثنائية الأبعاد يجب وجود بردم برنامج اوتوديسك مايا لاستخدام مشغلات الريندر الخاصة به من داخل برنامج التون بوم هارموني برمييم للحفاظ علي

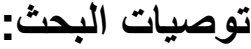

1 - استخدام برنامج تون بوم هارموني برميوم Toon Boom Harmony premium في انتاج أفلام رسوم متحركة ثنائية الأبعاد مدمجة بعناصر متحركة ثلاثية الأبعاد 2 - استخدام كامير ا برنامج تون بوم هارموني برميوم Toon Boom Harmony premium في اخر اج وتصوير المشاهد ثنائية الأبعاد المدمجة بعناصر متحركة ثلاثية الأبعاد

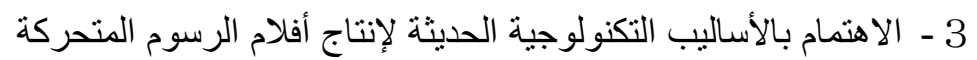

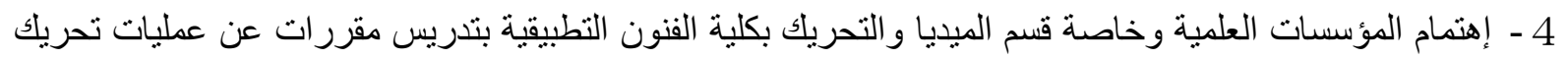
وإخر اج مشاهد ثنائية الأبعاد مدمجة بعناصر متحركة ثلاثية الأبعاد 5 - إهتمام المؤسسات الإنتاجية وشركات إنتاج الرسوم المتحركة بأهمية دمج الرسوم المتحركة ثنائية الأبعاد مدمجة بعناصر متحركة ثلاثية الأبعاد لما توفره من وقت وجودة وعمق فني باللقطات

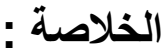
ان تحريك الرسومات ثنائية الأبعاد والمجسمات ثلاثية الأبعاد ودمجها داخل فراغ ثلاثي الأبعاد و إضافة الكاميرا المتحركة لربط المشهد و التعامل مع كافة الرسوم والمجسمات المتحركة داخل نفس المشهد يعد مؤخر ا مطلبا أساسيا لكافة السيناريوهات والرؤية الإخراجية لإنتاج أفلام الرسوم المتحركة مما بستدعي تحديد أفضل الطرق وأنسب الوسائل والأساليب الواجب استخدامها لمراحل الانتاج للفيلم المتحرك مع كيفية دمج وربط كل من الرسومات ثنائية الأبعاد

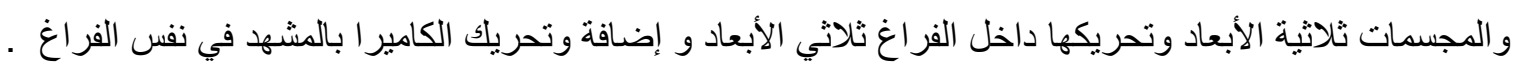
وباستخدام برنامج نون بوم هارموني برميوم Toon Boom Harmony premium في انتاج أفلام رسوم متحركة

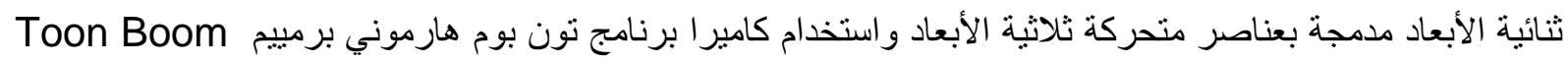
في اخر اج وتصوير المشاهد ثنائية الأبعاد المدمجة بعناصر متحركة ثلاثية الأبعاد تعد أفضل برل مئل الأساليب التكنولوجية الحديثة لإنتاج أفلام الرسوم المتحركة مع إمكانية محاكاة خصائص الكامير الما يوفره بداية من الرسم والتلوين والتحريك للرسوم ثنائية الأبعاد من ادارج التصاميم ثلاثية الأبعاد بنفس المشهد ثم إضافة الكاميرا و تحريكا للمحتوي ككل دون فقد أي عنصر ربط أو محاكاة أو تو افق حركة بسبب تنفيذ كافة العمليات داخل برنامج واحد . 
1 - Keith Osborn - Cartoon Character Animation with Maya: Mastering the Art of Exaggerated Animation - Bloomsbury - 2015

2 - Jeremy Hanke - Greenscreen Made Easy: Keying and Compositing Techniques for Indie Filmmakers - Michael Wiese Productions; 2nd edition (2009)

3 - https://www.toonboom.com/resources/white-papers 2 nov 2018

4 - Harold Whitaker - John Halas , Timing for Animation - Focal Press $-2^{\text {nd }}$ Edition -2009

5 - Angie Jones and Jamie Oliff, Thinking Animation - bringing the Gab between 2D and CG, Thomson, 2007

6 - https://www.toonboom.com/learn 2 oct 2018

7 - Peter Lord - Cracking Animation: The Aardman Book of 3-D Animation (Fourth edition) - Thamas a Hudson - 2015

8 - Chad Perkins -The After Effects Illusionist: All the Effects in One Complete Guide Focal Press - 2009

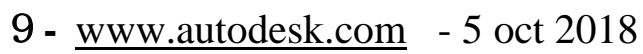

10 - Andy Beane - 3D Animation Essentials - Sybex - 2012

11 - Compositing Visual Effects Essentials for the Aspiring Artist - Steve Wright - Focal Press - Copyright (C) 2008

12 - https://www.toonboom.com/products/harmony 1 nov 2018 